\title{
SI - Rational Design of Chymotrypsin Inhibitor 2 by Optimizing Non-Native Interactions
}

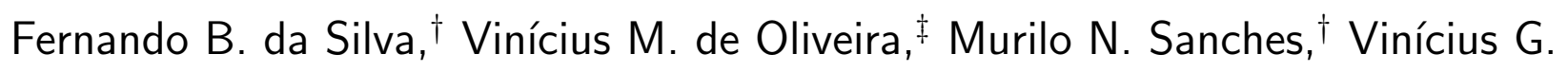
Contessoto, ${ }^{\top}, \S$ and Vitor B. P. Leite ${ }^{*, \dagger}$

$\dagger$ †epartment of Physics, São Paulo State University (UNESP), Institute of Biosciences, Humanities and Exact Sciences, São José do Rio Preto, SP, 15054-000, Brazil

$\ddagger$ Brazilian Biosciences National Laboratory, National Center for Research in Energy and Materials, LNBio/CNPEM, Campinas, SP, 13083-970, Brazil

IBrazilian Biorenewables National Laboratory - LNBR, Brazilian Center for Research in Energy and Materials - CNPEM, Campinas, SP, Brazil

$\S$ Center for Theoretical Biological Physics, Rice University, Houston, TX 77005, United States

E-mail: vitor.leite@unesp.br 


\section{Structure-Based C $\alpha$ Model}

The SBM potential is given by a harmonic term for the bonds and other one for the angles. The contact term is a Lennar-Jones type potential (with 10-12 instead of 6-12) and the noncontact term takes into account the repulsion interactions between pair of residues which are not in contact in the native structure. ${ }^{1-5}$

$$
\begin{aligned}
V\left(\Gamma, \Gamma_{o}\right) & =\sum_{\text {bonds }} \epsilon_{r}\left(r-r_{o}\right)^{2}+\sum_{\text {angles }} \epsilon_{\theta}\left(\theta-\theta_{o}\right)^{2} \\
& +\sum_{\text {backbone }} \epsilon_{\phi}\left\{\left[1-\cos \left(\phi-\phi_{o}\right)\right]+\frac{1}{2}\left[1-\cos \left(3\left(\phi-\phi_{o}\right)\right)\right]\right\} \\
& +\sum_{\text {contacts }} \epsilon_{C}\left[5\left(\frac{d_{i j}}{r_{i j}}\right)^{12}-6\left(\frac{d_{i j}}{r_{i j}}\right)^{10}\right]+\sum_{\text {non-contacts }} \epsilon_{N C}\left(\frac{\sigma_{N C}}{r_{i j}}\right)^{12}
\end{aligned}
$$

where $r$ is defined by the distance between two subsequent residues, $\theta$ is defined by the angles formed by three subsequent residues and $\phi$ is defined by the dihedral angles formed by four subsequent residues, while the subscript zero indicates that the parameter is taken from the native structure. The $d_{i j}$ is the native distante between the pair of contact $i$ and $j$, determined by the algorithm Shadow Contact Map. ${ }^{6}$ The parameters $\epsilon_{r}, \epsilon_{\theta}$ and $\epsilon_{\phi}$ describes the strenght of the bonds, angles and dihedral angles, respectively, with $\epsilon_{r}=100 \epsilon_{C}, \epsilon_{\theta}=$ $20 \epsilon_{C}, \epsilon_{\phi}=\epsilon_{C}$ and $\epsilon_{N C}=\epsilon_{C}$, with $\epsilon_{C}$ equal to 1 unit (reduced units) and $r_{i j}$ represents the distance between two non-covalent beads. 


\section{Electrostatic Free Energy of Engineering CI2}
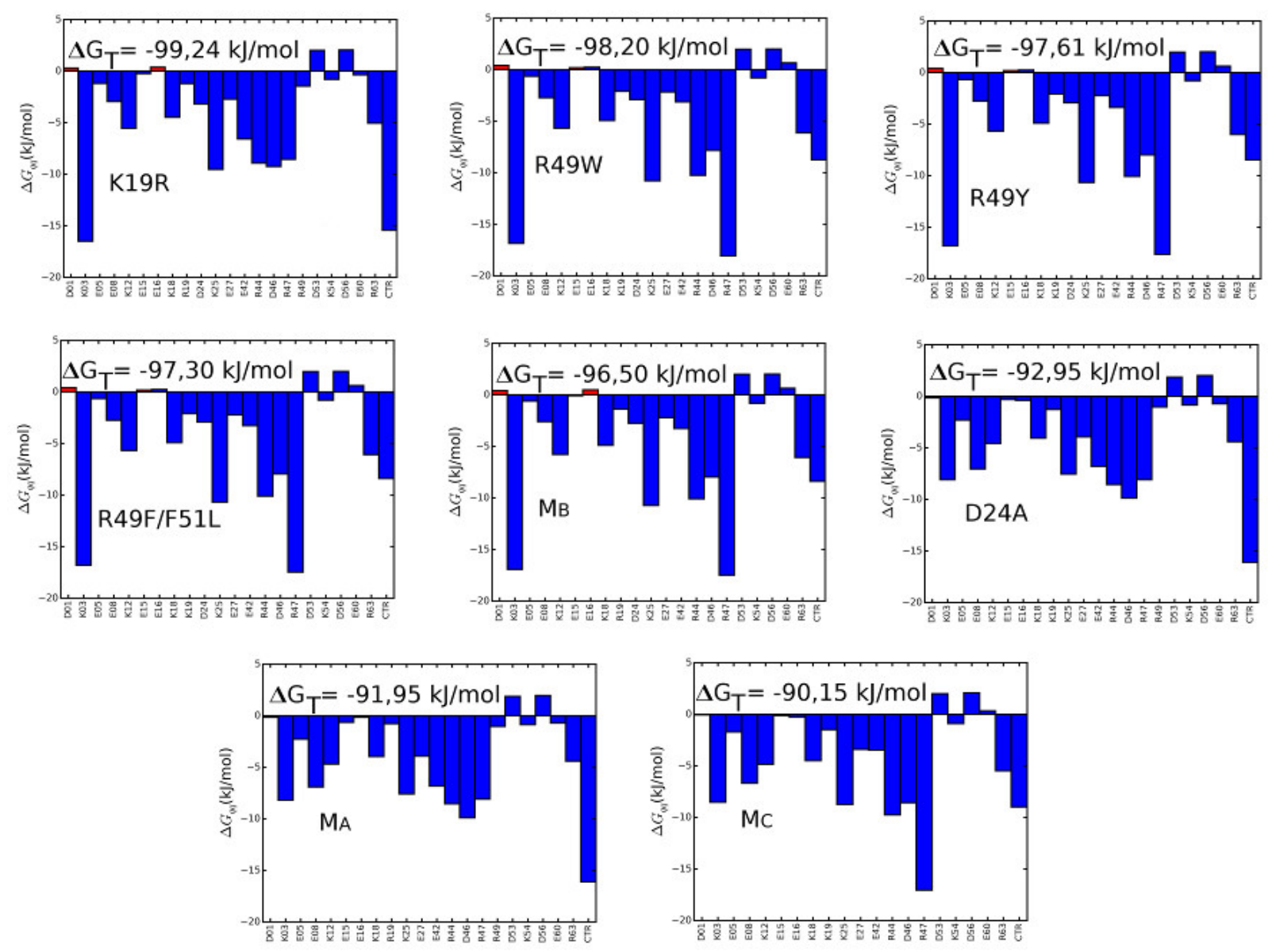

Figure S1: Electrostatic free energy profiles of each ionizable residue for all the engineered CI2. The following sequence of the mutants goes from the most to the leas thermostable: K19R, R49W, R49Y, R49F/F51L, M - K19R/R49F/F51L, D24A, M - K19R/D24A, M ${ }_{C}$ - D24A/R49F/F51L. The results were obtained in $\mathrm{pH}$ 7.0. The residues with SASA higher than $50 \%$ have a $\Delta \mathrm{G}_{\mathrm{qq}}>0$ and positive electrostatic free energy. 


\section{References}

(1) Clementi, C.; Nymeyer, H.; Onuchic, J. N. Topological and energetic factors: what determines the structural details of the transition state ensemble and "en-route" intermediates for protein folding? An investigation for small globular proteins. J. Mol. Biol. 2000, 298, 937-953.

(2) Sun, Y.; Ming, D. Energetic Frustrations in Protein Folding at Residue Resolution: A Homologous Simulation Study of Im9 Proteins. PLoS One 2014, 9, e87719.

(3) Contessoto, V. G.; Lima, D. T.; Oliveira, R. J.; Bruni, A. T.; Chahine, J.; Leite, V. B. P. Analyzing the effect of homogeneous frustration in protein folding. Proteins: Struct., Funct., Bioinf. 2013, 81, 1727-1737.

(4) de Mendonca, M. R.; Rizzi, L. G.; Contessoto, V.; Leite, V. B. P.; Alves, N. A. Inferring a weighted elastic network from partial unfolding with coarse-grained simulations. Proteins: Struct., Funct., Bioinf. 2014, 82, 119-129.

(5) Tripathi, S.; Garca, A. E.; Makhatadze, G. I. Alterations of Nonconserved Residues Affect Protein Stability and Folding Dynamics through ChargeCharge Interactions. J. Phys. Chem. B 2015, 119, 13103-13112.

(6) Noel, J. K.; Whitford, P. C.; Onuchic, J. N. The shadow map: a general contact definition for capturing the dynamics of biomolecular folding and function. J. Phys. Chem. B 2012, $116,8692-8702$. 